\title{
Introduction topical issue on CT plaque burden
}

\author{
Johan H. C. Reiber ${ }^{1}$ \\ Published online: 9 October 2020 \\ (c) Springer Nature B.V. 2020
}

It has taken some time to put this Topical Issue together, but I believe that we do have now a very nice and very upto-date collection of Review and Overview papers on the various topics associated with the very interesting and timely field of the CT Plaque Burden. Before we begin, I would like to thank all the authors who have contributed to this nice Topical Issue.

This Topical Issue starts with a Review on the topic of serial CTA to assess plaque progression and the therapeutic effects of anti-atherosclerotic drugs by Jana Taron et al. of the group of Udo Hoffmann at Mass General in Boston, USA [1]. This team has a long history of research in this field. Because CTA is noninvasive, it allows for setting up and enabling clinical trials with lower-risk populations, higher retention rates, and lower costs. For these important reasons they see an increasing interest and usage of serial CTA as an imaging endpoint in clinical trials.

The next review paper by Parastou Eslami is from the same group [2]. Given the high spatial and temporal resolution of the modern CT scanners, it is now possible to assess the presence, morphology and composition of coronary atherosclerosis and derive high risk features from the Plaque Burden analyses, such as large plaque volume, low CT attenuation, napkin-ring sign, spotty calcification and positive remodeling. However, in this overview the group examines the value of lesion-specific hemodynamics, such as endothelial shear stress, fractional flow reserve and plaque stress. They believe that this combination of anatomic and hemodynamic information allows the identification of lesions at risk for rupture earlier than only on the basis of anatomic information.

Dr Suvanini Lakshmanan of the team of Matthew $\mathbf{J}$ Budoff in Torrance, California, USA wrote a review about the benefits of imaging and plaque analysis in serial coronary CTA for the evaluation of cardiovascular risk analysis

Johan H. C. Reiber

J.H.C.Reiber@lumc.nl

1 Leiden University Medical Center, Leiden, The Netherlands and targeted treatment of coronary artery disease [3]. They believe that whole-heart quantification and characterization of coronary atherosclerotic plaque burden on CTA allows the transition form identifying a "vulnerable" plaque to the "vulnerable" patient.

Dr Nestor Gahungu from the University of Ottawa Heart Institute, Canada under the leadership of Dr Benjamin J Chow and Dr Girish Dwivedi studied the differences in plaque scores between a South Asian population and matched Caucacian population [4]. They found no differences in low, intermediate, and high-attenuation or calcified normalized plaque volumes, and after a follow-up period of 32 months, the major adverse cardiovascular events were also similar. Since South Asian populations experience poorer cardiovascular outcomes in general, other factors need to be investigated.

In the next paper by Dr Sang-Eun Lee together with many co-authors from multiple centers, all under the supervision of dr Hyuk-Jae Chang from Yonsei University College of Medicine, Seoul, Korea investigated whether the assessment of individual plaques is superior in predicting the progression of coronary artery disease than a per-patient analysis [5]. From a total of 1297 patients and 3218 non-obstructive lesions in the PARADIGM trial, who underwent $a \geq 2$-year follow-up with CTA, they concluded that the lesion-level analysis of coronary atherosclerotic plaques yielded a better predictive power for the development of obstructive CAD than the simple quantification of total coronary atherosclerotic burden at a patient-level.

Dr Satoru Kishi under the leadership of Dr Armin ArbabZadeh at Johns Hopkins University in Baltimore, USA, followed a total of 372 patients with suspected or known CAD, who were enrolled in the CORE320 Trial, for a period of 2 years after baseline CTA and invasive coronary angiography (QCA) [6]. The purpose of the study was to provide prognostic information about plaque volume and stenosis assessment in these patients. They found that angiographic and plaque metrics performed only modestly in the prediction of 30-day revascularization and 2-year MACE in 
high-risk patients, and these improved after the exclusion of patients with known CAD.

In a study by the Medstar Washington Hospital Center, USA, Dr Nausharwan Butt and colleagues under the supervision by Dr Hector Garcia-Garcia, the investigators compared the CT Leaman and Leiden scores in overweight and obese populations [7]. In general, these two scores utilize coronary plaque location, composition and severity of stenosis to risk stratify patients for cardiovascular disease. It turned out in this study that the obese and overweight individuals have similar average CT Leaman and Leiden scores, but the obese patients were more likely to have higher CT Leiden scores, which may indicate a higher risk for adverse cardiovascular outcomes.

In a second study by the Medstar Washington Hospital Center, USA, Dr Alexandre Hideo-Kajita studied the possible use of the CT-adapted Leaman score (CT-LeSc) and the CT Liver and Spleen Attenuation (CT-LSA)score in patients with non-alcoholic fatty liver disease (NAFLD) and to characterize the inflammatory processes of each disease state [8]. They concluded that some CT-LSA parameters correlate with high risk CT-LeSc and may provide complementary information for cardiovascular risk stratification. Also, the significant metrics of liver absolute attenuation value and liver and spleen attenuation difference can be quickly completed in the clinical setting and may support a suspicion of CAD.

In another study by Dr Giovanni Monizzi and other coauthors from multiple sites, under the supervision of Dr Carlos Collet at OLV Cardiovascular Center in Aalst, Belgium, they compared the coronary artery calcifications by CT with OCT intravascular imaging in 66 calcified plaques from 32 vessels in 31 patients [9]. The coronary CT and OCT images were matched using fiduciary points. They found that the coronary $\mathrm{CT}$ volumetric calcium overestimates calcium volume by $60 \%$ compared to OCT, although there were no differences in minimal lumen areas (MLAs). They conclude that coronary CT may emerge as a tool to quantify calcium burden for invasive procedural planning, if the proper corrections are carried out.

This Topical Issue would not be complete, if we also had not submissions about the value and expectations of Artificial Intelligence (AI) and Machine Learning (ML) in this field. The first paper is by Dr Diana Opincariu under the supervision of Dr Theodora Benedek of the University of Medicine "George Emil Palade" in Romania [10]. The team provide an overview of the current evidence on the complex role of CTA in the detection and quantification of vulnerable plaques and coronary inflammation, and the most recent results of the radiomics-based machine learning for the complex assessment of plaque-associated risks.

In the next paper by Dr Verena Brandt of the Medical University of South Carolina under the leadership of Dr
Joseph Schoepf and together with co-authors from multiple centers in Germany [11]. The goal of this review paper is to provide an overview about the contemporary state of Machine Learning based algorithms in cardiac CT, and to provide clinicians with currently available scientific data on the clinical validation and implementation of these algorithms for the prediction of ischemia-specific CAD and cardiovascular outcome. Of course, we are still at the very beginning, but ML offers a powerful platform to integrate clinical and imaging data for improved patient care.

Finally, we also see entrance of the CT coronary plaque assessment into the cath lab, which is nicely summarized in the last paper by Dr Breda Hennessey from Hospital Clinico San Carlos in Madrid, Spain under the supervision of Dr Javier Escaned [12]. As of yet, coronary computed tomography angiography (CCTA) is still ignored by most interventional cardiologists as a tool in the planning of PCI strategies. However, in the future, many more patients referred to the cath lab will have had a prior CCTA study. In this review article, the authors will revisit the most frequent scenarios of complex PCI and establish analogies between the use of intracoronary diagnostics and CCTA in setting the procedural strategy, and in anticipating specific challenges.

\section{References}

1. Taron J, Lee S, Aluru J et al (2020) A review of serial coronary computed tomography angiography (CTA) to assess plaque progression and therapeutic effect of anti-atherosclerotic drugs. Int J Cardiovasc Imaging. https://doi.org/10.1007/s10554-020-01793 -w

2. Eslami P, Thondapu V, Karady J et al (2020) Physiology and coronary artery disease: emerging insights from computed tomography imaging based computational modeling. Int J Cardiovasc Imaging. https://doi.org/10.1007/s10554-020-01954-X

3. Lakshmanan S, Rezvanizadeh V, Budoff MJ (2020) Comprehensive plaque assessment with serial coronary $\mathrm{CT}$ angiography: translation to bedside. Int J Cardiovasc Imaging. https://doi. org/10.1007/s10554-020-01849-x

4. Gahungu N, Tewari S, Liu YW et al (2020) Quantified coronary plaque characteristics between Caucasian and Morise scorematched South Asian populations. Int J Cardiovasc Imaging. https ://doi.org/10.1007/s10554-020-01802-y

5. Lee S, Sung JM, Andreini D et al (2020) Per-lesion versus perpatient analysis of coronary artery disease in predicting the development of obstructive lesions: the Progression of AtheRosclerotic PlAque DetermIned by Computed TmoGraphic Angiography Imaging (PARADIGM) study. Int J Cardiovasc Imaging. https:// doi.org/10.1007/s10554-020-01960-Z

6. Kishi S, Magalhães TA, Cerci RJ et al (2020) Comparative effectiveness of coronary artery stenosis and atherosclerotic plaque burden assessment for predicting 30-day revascularization and 2 -year major adverse cardiac events. Int J Cardiovasc Imaging. https://doi.org/10.1007/s10554-020-01851-3

7. Butt N, Parajuli S, Ali L et al (2020) Comprehensive assessment of coronary computed tomography angiography by using Leaman and Leiden score in overweight and obese patients. Int $\mathbf{J}$ 
Cardiovasc Imaging. https://doi.org/10.1007/s10554-020-01938 $-\mathrm{X}$

8. Hideo-Kajita A, Garcia-Garcia HM, Wopperer SB et al (2020) Correlation between computed tomography adapted leaman score and computed tomography liver and spleen attenuation parameters for non-alcoholic fatty liver disease as well as respective inflammatory mediators. Int J Cardiovasc Imaging. https://doi. org/10.1007/s10554-020-02026-w

9. Monizzi G, Sonck J, Nagumo S et al (2020) Quantification calcium burden by coronary CT angiography compared to optical coherence tomography. Int J Cardiovasc Imaging. https://doi. org/10.1007/s10554-020-01839-z

10. Opincariu D, Benedek T, Chiu M et al (2020) From CT to artificial intelligence for complex assessment of plaque-associated risk. Int J Cardiovasc Imaging. https://doi.org/10.1007/s10554-020-01926 $-1$
11. Brandt V, Emrich T, Schoepf UJ et al (2020) Ischemia and outcome prediction by cardiac CT based machine learning. Int J Cardiovasc Imaging. https://doi.org/10.1007/s10554-020-01929-y

12. Hennessey B, Vera-Urquiza R, Mejía-Rentería H et al (2020) Contemporary use of coronary computed tomography angiography in the planning of percutaneous coronary intervention. Int J Cardiovasc Imaging. https://doi.org/10.1007/s10554-020-02052-8

Publisher's Note Springer Nature remains neutral with regard to jurisdictional claims in published maps and institutional affiliations. 